\title{
THE CONSTRUCTION OF BRANCHED COVERING RIEMANN SURFACES
}

\author{
by R. A. RANKIN \\ (Received 8th April, 1958)
}

1. Introduction. In some recent work on uniformization [2], I found it necessary to consider a regular branched covering Riemann surface $\hat{R}$ of a given Riemann surface $R_{f}$, where $R_{f}$ is an unlimited branched, but not necessarily regular, covering surface of a portion $R_{z}$ of the extended complex $z$-plane $Z$ (2-sphere). The branching of $\hat{R}$ over $R_{f}$ had to be chosen so that $\hat{R}$ was regular over $R_{z}$, since the uniformization of the functions on $R_{f}$ is then simpler ; in particular, the Schwarzian derivative is then a single-valued function of $z$.

Although branched covering surfaces are considered in varying detail in different books on topology, Riemann surfaces and uniformization, I have been unable to find any place where the construction of such surfaces, possessing branch points of preassigned orders, is described, with the exception of the work of Fourès [1], which applies only to closed Riemann surfaces. The object of this paper is to show how such branched covering surfaces can be constructed. The method used is based on a standard method for constructing smooth covering surfaces, as described in § 4-3 of Springer's book [3], and the terminology used is the same.

Let $R$ be a given Riemann surface, and let $B$ be a set of isolated points on it. We write $S=R-B$ and denote by $F$ and $G$ the fundamental homotopy groups of $R$ and $S$, respectively, relative to a fixed base point $A \in S$. Let $\hat{R}$ be an unlimited covering surface of $R$ which is smooth except possibly at points $\hat{Q}$ lying over the points $Q$ of $B$, and denote by $\hat{S}$ the surface obtained from $\hat{R}$ by omitting all such points. Then $\hat{S}=\hat{R}-\hat{B}$, where $\hat{B}$ is the set of all such points $\hat{Q}$. Let $\hat{A}$ be any fixed point of $\hat{S}$ lying over $A$. B is always assumed closed in $R$.

Then $\hat{S}$ is a smooth unlimited covering surface of $S$, and we denote by $\hat{F}$ and $\hat{G}$ the homotopy groups of $\hat{R}$ and $\hat{S}$, respectively. Let $i$ and $\hat{\imath}$ denote the inclusion mappings from $\mathcal{S}$ into $R$ and from $\hat{S}$ into $\hat{R}$, respectively, and let $p$ be the projection mapping from $\hat{R}$ onto $R$, and from $\hat{S}$ onto $S$ (see diagram).

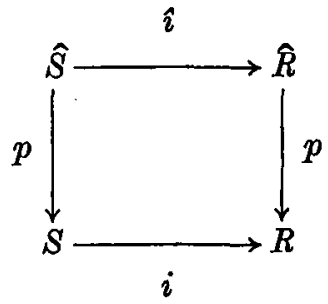

We also define two epimorphisms

by

$$
\phi: G \rightarrow F, \text { and } \hat{\phi}: \hat{\theta} \rightarrow \hat{F},
$$

$$
\phi(\{C\})=\{i(C)\}, \text { and } \hat{\phi}(\{\hat{C}\})=\{\hat{\imath}(\hat{C})\} \text {. }
$$

Here $C$ and $\hat{C}$ are closed curves in $S$ and $\hat{S}$, respectively, which are drawn from the base points $A$ and $\hat{A}$, and homotopy classes are denoted by $\{\ldots\}$. We also write 


$$
\operatorname{Ker} \phi=H \text { and } \operatorname{Ker} \hat{\phi}=\hat{H} \text {, }
$$

for the kernels of these epimorphisms. Thus $H$ is the subgroup of $G$ consisting of classes $\{C\}$ of curves in $S$ which are homotopic to a constant in $R$; similarly, $\hat{H}$ is the subgroup of $\hat{G}$ consisting of classes $\{C\}$ of curves in $\hat{S}$ which are homotopic to a constant in $\hat{R}$. We note that, since open disks round points of $B$ and $\hat{B}$ are simply connected in $R$ and $\hat{R}$, we need only consider curves $C$ and $\hat{C}$ which do not pass through points of $B$ and $\hat{B}$, respectively. The subgroups $H$ and $\hat{H}$ are normal in $G$ and $\hat{G}$, respectively, and

$$
F \cong G / H, \quad \hat{F} \cong \hat{G} / \hat{H} \text {. }
$$

The mapping $\{\hat{C}\} \rightarrow\{p(\hat{C})\}$ from $\hat{G}$ into $G$ is an isomorphism of $\hat{G}$ onto a subgroup of $G$; this follows from the monodromy theorem. We can therefore regard $\hat{G}$, and consequently $\hat{H}$, as subgroups of $G$. On the other hand, the same mapping from $\hat{F}^{\prime}$ into $F^{\prime}$ is, in general, only a homomorphism of $\hat{F}$ onto a subgroup of $F$; it is easily seen that this homomorphism is an isomorphism if and only if $\hat{G} \cap H=\hat{H}$.

The covering surface $\hat{S}$ is said to be regular over $S$ if $\hat{G}$ is normal in $G$, and then $\hat{R}$ is also said to be regular over $R$. We are particularly interested in regular simply connected branched covering surfaces $\hat{R}$ over a given surface $R$. The groups $\hat{G}$ and $\hat{H}$ then coincide and are normal subgroups of $G$.

2. The subgroups $H$ and $\hat{H}$. To each point $Q \in B$ we make correspond a fixed open disk $N_{Q}$ in $R$ about $Q$ as centre, and suppose that, in one system of local coordinates $z=\Phi(P)\left(P \in N_{Q}\right), N_{Q}$ is given by $|z|<1$ and $\Phi(Q)=0$. We suppose that no two of the disks $N_{Q}$ overlap. Let $Q^{\prime}$ be the point $z=\frac{1}{2}$ in $N_{Q}$, and write $\gamma_{Q}$ for the closed curve in $N_{Q}$, which is given by

$$
z=\frac{1}{2} e^{2 \pi i t} \quad(0 \leqslant t \leqslant 1) .
$$

For any curve $\alpha_{Q}$ joining $A$ to $Q^{\prime}$ in $S$, we write†

$$
C\left(\alpha_{Q}\right)=\alpha_{Q} \gamma_{Q} \alpha_{Q}^{-1} \text {. }
$$

Then $H$ is the subgroup of $G$ generated by the classes $\left\{C\left(\alpha_{Q}\right)\right\}$ for all $\alpha_{Q}$ and all $Q \in B$.

Now let $\hat{Q}$ be any point of $\hat{R}$ lying over a point $Q \in B$, and let it be a branch point of order $q(\hat{Q})-1$. We may suppose that the local coordinate $\hat{z}$ in the open disk $\hat{N}_{Q}$ covering $N_{Q}$ and containing $\hat{Q}$ is given by $z=\hat{z}^{q}$, where $q=q(\hat{Q})$. There will be $q(\hat{Q})$ different points in $\hat{N}_{Q}$ lying over $Q^{\prime}$. Select any one of these and call it $\hat{Q}^{\prime}$. Then we can choose $\alpha_{Q}$ so that the curve $\hat{\alpha}_{Q}$, which lies over it in $\hat{S}$ and has initial point $\hat{A}$, terminates at $\hat{Q}^{\prime}$. With this $\alpha_{Q}$, write

so that

$$
C^{*}\left(\alpha_{Q}\right)=C^{q}\left(\alpha_{Q}\right), \quad \text { where } q=q(\hat{Q}),
$$

$$
\left\{C^{*}\left(\alpha_{Q}\right)\right\}=\left\{\alpha_{Q} \gamma_{Q}^{q} \alpha_{Q}^{-1}\right\} \quad(q=q(\hat{Q})),
$$

in $S$. Since different choices of $\hat{Q}^{\prime}$ in $\hat{N}_{Q}$ can be obtained by replacing $\alpha_{Q}$ by $\alpha_{Q} \gamma_{Q}^{r}(0 \leqslant r<q)$, we see from (4) that $\left\{C^{*}\left(\alpha_{Q}\right)\right\}$ is independent of the particular choice of $\hat{Q}^{\prime}$ from the $q(\hat{Q})$ possible points in $\hat{N}_{Q}$ which lie over $Q^{\prime}$. Let $\hat{C}^{*}\left(\alpha_{Q}\right)$ lie over $C^{*}\left(\alpha_{Q}\right)$ in $\hat{S}$, relative to the base point $\hat{A}$. Then $\hat{C}^{*}\left(\alpha_{Q}\right)$ is closed in $\hat{S}$ and is homotopic to a constant in $\hat{R}$. The group $\hat{H}$ is

† Since we are only interested in homotopy classes we can ignore the fact that curve multiplication is not associative. 
the subgroup of $\hat{G}$ generated by the classes $\left\{\hat{C}^{*}\left(\alpha_{Q}\right)\right\}$; i.e. $\hat{H}$ is the subgroup of $G$ generated by the classes $\left\{C^{*}\left(\alpha_{Q}\right)\right\}$. We note also, for future reference, that, if $\left\{C\left(\alpha_{Q}\right)\right\}^{*} \in \hat{G}$, then $q(\hat{Q})$ divides $r$.

From this we deduce that $\hat{H}$ is a normal subgroup of $G$ when $\hat{R}$ is regular over $R$. For let $\Gamma$ be any closed curve in $S$ drawn from $A$ and put

so that

$$
\beta_{Q}=\Gamma \alpha_{Q}
$$

$$
\left\{\Gamma C\left(\alpha_{Q}\right) \Gamma^{-1}\right\}=\left\{C\left(\beta_{Q}\right)\right\} .
$$

Curves $\hat{\alpha}_{Q}$ and $\hat{\beta}_{Q}$ drawn from $\hat{A}$ in $\hat{S}$ over $\alpha_{Q}$ and $\beta_{Q}$ will terminate at points $\hat{Q}_{1}^{\prime}$ and $\hat{Q}_{2}^{\prime}$ over $Q^{\prime}$, respectively, and so will determine points $\hat{Q}_{1}$ and $\hat{Q}_{2}$ lying over $Q$. Also any two such points can be determined in this way. Let

$$
q_{1}=q\left(\hat{Q}_{1}\right), \quad q_{2}=q\left(\hat{Q}_{2}\right) .
$$

Since $\hat{G}$ is normal in $G$ and $\left\{C^{q_{1}}\left(\alpha_{Q}\right)\right\} \in \hat{G}$, we deduce from (5) that $\left\{C^{q_{1}}\left(\beta_{Q}\right)\right\} \in \hat{G}$. But $q_{2}$ is the least positive integer with the property that $\left\{C^{q_{3}}\left(\beta_{Q}\right)\right\} \in \hat{G}$, and we deduce that $q_{2}$ divides $q_{1}$; similarly $q_{1}$ divides $q_{2}$, so that $q_{1}=q_{2}$. Accordingly $q(\hat{Q})$ takes the same value $q(Q)$, say, for all points $\hat{Q}$ lying over $Q$, and we deduce that $\hat{H}$ is normal in $Q$, since it is generated by the classes $\left\{C^{Q(Q)}\left(\alpha_{Q}\right)\right\}$.

3. Construction of $\hat{R}$ over $R$. In this section we construct a regular branched covering Riemann surface $\hat{R}$ over a given Riemann surface $R$. For each point $Q$ belonging to a set $B$ of isolated points of $R$ we take an integer $q(Q)>1$, our intention being that $\hat{R}$ shall have branch points of order $q(Q)-1$ at all points lying over $Q$.

The surface $S$ and the associated groups $G$ and $H$ are defined as previously. We denote by $H^{\prime}$ the normal subgroup of $G$ and $H$ which is generated by the classes $\left\{C^{a(\theta)}\left(\alpha_{Q}\right)\right\}$, and take $G^{\prime}$ to be any normal subgroup of $G$ which contains $H^{\prime}$ and is such that

$$
\left\{C^{r}\left(\alpha_{Q}\right)\right\} \in G^{\prime} \text { implies that } q(Q) \text { divides } r \text {. }
$$

The relevance of this condition, which affects the choice of the numbers $q(Q)$ as well as the group $G^{\prime}$, will appear later. We note here that it does not necessarily follow from (6) that $G^{\prime} \cap H=H^{\prime}$.

We now construct covering manifolds $\hat{R}$ and $\hat{S}$ over $R$ and $S$, where $\hat{R}$ is branched over $R$ and $\hat{S}$ is smooth over $S$. We shall show that the branch points of $\hat{R}$ over $R$ are of order $q(Q)-1$ at points lying over points $Q$ of $B$, and that the groups $\hat{G}$ and $\hat{H}$ so determined satisfy $\widehat{G}=G^{\prime}$ and $\hat{H}=H^{\prime}$.

A point $\hat{P}$ of $\hat{S}$ lying over a point $P$ of $S$ is defined to be an equivalence class $\{P, C\}$ of pairs $(P, C)$, where $C$ is a curve in $S$ joining the base point $A$ to $P$. For this purpose, two pairs $\left(P, C_{1}\right)$ and $\left(P, C_{2}\right)$ are defined to be equivalent if and only if

$$
\left\{C_{1} C_{2}^{-1}\right\} \in G^{\prime} \text {. }
$$

This is clearly an equivalence relation. There is therefore a one-to-one correspondence between the points $\hat{P}$ of $\hat{S}$ lying over $P$ and the elements of $G / G^{\prime}$.

To obtain $\hat{R}$, we add to $\hat{S}$ a set $\hat{B}$ of points lying over the points of $B$. A point $\hat{Q}$ of $\hat{R}$ lying over a point $Q$ of $B$ is defined to be an equivalence class $\left\{Q, \alpha_{Q}\right\}$ of pairs $\left(Q, \alpha_{Q}\right)$, where $\alpha_{Q}$ joins $A$ to $Q^{\prime}$ in $S$. Here two pairs $\left(Q, \alpha_{Q}\right)$ and $\left(Q, \beta_{Q}\right)$ are defined to be equivalent if and 
only if

$$
\left\{\alpha_{Q} \gamma_{Q}^{r} \beta_{Q}^{-1}\right\} \in Q^{\prime} \text { for some integer } r \text {. }
$$

This is clearly an equivalence relation. Note that the equivalence classes $\left\{Q, \alpha_{Q} \gamma_{Q}^{\dagger}\right\}$ define the same point $\hat{Q}$ for all integers $r$.

The projection mapping $p$ from $\hat{R}$ to $R$ is defined by

$$
p:\{P, C\} \rightarrow P \quad(P \in S), \quad\left\{Q, \alpha_{Q}\right\} \rightarrow Q \quad(Q \in B) .
$$

We now introduce a topology in $\hat{R}$ and $\hat{S}$ by defining open disks and then open sets. Let $\hat{P}_{0}=\left\{P_{0}, C_{0}\right\}$ be a point of $\hat{S}$, and let $N$ be any open disk in $S$ which is centred at $P_{0}$. An open disk $\hat{N}$ about $\hat{P}_{0}$ in $\hat{S}$ or $\hat{R}$ is defined to be the set of all points $\hat{P}=\left\{P, C_{0} J\right\}$ of $\hat{S}$ such that $P \in N$, where $J$ is any curve lying in $N$ which joins $P_{0}$ to $P$. Since $N$ is simply connected, two such curves $J_{1}$ and $J_{2}$ are homotopic and so the definition of $\hat{N}$ is independent of the particular curves $C_{0}$ and $J$ chosen to represent $\hat{P}_{0}$ and $\hat{P}$. We note that the mapping $p$ is one-to-one from $\hat{N}$ to $N$ and that the same local coordinates can be taken in $\hat{N}$ and $N$.

Now suppose that $\hat{Q}=\left\{Q, \alpha_{Q}\right\}$ is a point of $\hat{B}$, and let $N$ be an open disk in $R$ which is centred at $Q$ and is contained in $N_{Q}$. An open disk $\widehat{N}$ in $\widehat{R}$ about $\widehat{Q}$ is defined to consist of $\hat{Q}$ and all points $\hat{P}=\left\{P, \alpha_{Q} J\right\}$ of $\hat{S}$ such that $P \in N$ and $J$ is any curve in

$$
N_{Q}^{\prime}=N_{Q}-Q
$$

joining $Q^{\prime}$ to $P$. In this case, different choices of $J$ may yield different points $\hat{P}$ lying over the same point $P \in N$, since $N_{Q}^{\prime}$ is not simply connected. This definition is independent of the choice of $\alpha_{Q}$ in the pair $\left(Q, \alpha_{Q}\right)$ representing $\hat{Q}$; for if $\hat{Q}=\left\{Q, \beta_{Q}\right\}$ where (8) holds, then, by (7), $\left\{P, \alpha_{Q} J\right\}=\left\{P, \beta_{Q} J^{\prime}\right\}$ where $J^{\prime}=\gamma_{Q}^{-r} J$.

A set $\hat{V}$ of points of $\hat{S}$ or $\hat{R}$ is defined to be open if each point of $\hat{V}$ is contained in an open disk which is contained entirely in $\hat{V}$. These open sets define a topology in $\hat{S}$ and $\hat{R}$. To show that this is a Hausdorff topology we must show that disjoint open sets can be described about any two different points. This is obvious when the projections of the two points in $R$ are different. The other cases are not so immediate although they are straightforward; we give a proof for the hardest case only, when the two points $\hat{Q}_{1}$ and $\hat{Q}_{2}$ are different points of $\hat{B}$ lying over the same point $Q \in B$.

Let $\hat{Q}_{1}=\left\{Q, \alpha_{Q}\right\}$ and $\hat{Q}_{2}=\left\{Q, \beta_{Q}\right\}$, where,

$$
\text { for all integers } r,\left\{\alpha_{Q} \gamma_{Q}^{r} \beta_{Q}^{-1}\right\} \notin G^{\prime} \text {. }
$$

Let $\hat{N}_{1}$ and $\hat{N}_{2}$ be open disks about $\hat{Q}_{1}$ and $\hat{Q}_{2}$, respectively, which lie over an open disk $N$ about $Q$, where $N \subseteq N_{Q}$. Then $\hat{N}_{1}$ and $\hat{N}_{2}$ are disjoint ; for otherwise they would contain a common point

$$
\hat{P}=\left\{P, \alpha_{Q} J_{1}\right\}=\left\{P, \beta_{Q} J_{2}\right\}
$$

of $\hat{S}$, where the curves $J_{1}$ and $J_{2}$ join $Q^{\prime}$ to $P$ in $N_{Q}^{\prime}$, and so, by (7),

$$
\left\{\alpha_{Q} J_{1}\left(\beta_{Q} J_{2}\right)^{-1}\right\}=\left\{\alpha_{Q}\left(J_{1} J_{2}^{-1}\right) \beta_{Q}^{-1}\right\} \in G^{\prime} .
$$

But $J_{1} J_{2}^{-1}$ is homotopic in $S$ to $\gamma_{Q}^{*}$, for some integer $r$, and so (10) contradicts (9).

To show that $\hat{R}$ and $\hat{S}$ are manifolds, it remains to prove that they are connected. This is obvious for $\hat{S}$ and is also true for $\hat{R}$, since the point $\left\{Q, \alpha_{Q}\right\}$ can clearly be connected to 
$\left\{Q^{\prime}, \alpha_{Q}\right\}$. Further, every open disk $\hat{N}$ is simply connected. In fact, every closed curve in $\hat{N}$ is freely homotopic to the centre of $\hat{N}$.

With this topology, $p$ is a continuous mapping of $\hat{R}$ onto $R$ and is bicontinuous from $\hat{S}$ to $S$. Since the local coordinates can be taken to be the same in each pair of open disks $N$ and $\hat{N}$ centred at points of $S$ and $\hat{S}$, it follows that $\hat{S}$ is a smooth covering manifold of $S$ and is clearly unlimited. Also $\hat{R}$ is an unlimited covering manifold of $R$ which is smooth except possibly at points lying over the set $B$.

Let $\hat{Q}=\left\{Q, \alpha_{Q}\right\}$ be a point of $\hat{R}$ lying over $Q \in B$, and let $\hat{N}$ be an open disk about $\hat{Q}$ covering the open disk $N$ which is centred at $Q$, where $N \subseteq N_{Q}$. Two points $\left\{P, \alpha_{Q} J_{1}\right\}$ and $\left\{P, \alpha_{Q} J_{2}\right\}$ lying over a point $P \in N-Q$ are identical if and only if

$$
\left\{\alpha_{0} J_{1}\left(\alpha_{Q} J_{2}\right)^{-1}\right\}=\left\{\alpha_{0}\left(J_{1} J_{2}^{-1}\right) \alpha_{Q}^{-1}\right\} \in G^{\prime} .
$$

Since $J_{1} J_{2}^{-1}$ is homotopic in $S$ to $\gamma_{Q}^{r}$, for some integer $r$, this condition becomes, in the notation of (2), $\left\{C\left(\alpha_{Q}\right)\right\}^{r} \in G^{\prime}$. It follows from (6) that there are exactly $q(Q)$ different points of $\hat{N}$ which lie over $P$, namely the points

$$
\left\{P, \alpha_{Q} J \gamma_{Q}^{\top}\right\} \quad(r=0,1,2, \ldots, q(Q)-1),
$$

where $J$ is any curve joining $Q^{\prime}$ to $P$ in $N_{Q}^{\prime}$. Thus $\hat{Q}$ is a branch point of the correct order $q(Q)-1$ over $Q$. Local coordinates $\hat{z}$ and $z$ can be chosen in $\hat{N}$ and $N$ in terms of which the mapping $p$ is given by $z=\hat{z}^{q(Q)}$.

It follows from this and $\S 2$ that $\hat{H}=H^{\prime}$, and it remains to show that $\hat{G}=G^{\prime}$. Let $\hat{C}$ be any closed curve in $\hat{S}$ relative to the base point $\hat{A}=\{A, 1\}$, where 1 denotes the constant curve through $A$. The projection $C$ of $\widehat{C}$ in $S$ is a closed curve relative to the base point $A$. Since the end point $\hat{A}$ of $\hat{C}$ can also be represented by $(A, C)$, we have $\{C\} \in Q^{\prime}$. Conversely, if $\{C\} \in G^{\prime}$, then the curve $\hat{C}$ lying above it in $\hat{S}$ which begins at $\hat{A}$ also ends there ; thus $\hat{Q}=G^{\prime}$. The surfaces $\hat{R}$ and $\hat{S}$ are clearly Riemann surfaces.

It is easily seen that any other covering surface $\hat{R}^{\prime}$ of $R$ corresponding to the same groups $\hat{G}$ and $\hat{H}$ and with the same values of $q(Q)$, is homeomorphic to $\hat{R}$, so that $\hat{R}$ is unique to within a homeomorphism. Also every unlimited regular branched covering surface $\hat{R}$ over $R$ can be constructed in this way, since it was shown in $\S 2$ that the condition (6), with $G^{\prime}=\hat{G}$, is necessary as well as sufficient. We have therefore proved the following theorem.

TheOREM 1. Let $R$ be a Riemann surface containing a closed set $B$ of isolated points, and let an integer $q(Q)>1$ be assigned to each point $Q \in B$. Let $G$ be the fundamental group, relative to a fixed base point, of the surface $S=R-B$, and let $H$ be the subgroup of $G$ consisting of classes of curves in $S$ which are homotopic to a constant in $R$. Let $\hat{H}$ be the subgroup of $H$ generated by the classes $\left\{C^{*}\left(\alpha_{0}\right)\right\}$ of $(3)$, and let $\hat{G}$ be any normal subgroup of $G$ which contains $\hat{H}$ and satisfies the condition that, for all $Q \in B$,

Also, let $\hat{H}=\hat{G} \mid \hat{H}$.

$$
\left\{C\left(\alpha_{Q}\right)\right\}^{r} \in \hat{G} \text { implies that } q(Q) \text { divides } r \text {. }
$$

Then a regular unlimited branched covering Riemann surface $\hat{R}$ over $R$ can be constructed which has branch points of order $q(Q)-1$ at the points $\hat{Q}$ of $R$ which lie over the points $Q$ of $B$, and which has a fundamental group isomorphic to $\hat{H}$. If $\hat{B}$ is the set of all such points $Q$ and $\hat{S}=\hat{K}-\hat{B}$, then $\hat{S}$ is a regular unlimited smooth covering surface of $S$.

Conversely, every regular unlimited branched covering Riemann surface $\hat{R}$ over $R$ can be constructed in this way. 
As remarked earlier, the condition (11) may impose some restrictions on the choice of the integers $q(Q)$, and we now consider this for the most important case when $\hat{R}$ is simply connected, i.e. when we take $\hat{G}=\hat{H}$. The situation is similar in the more general case when

$$
\hat{G} \cap H=\hat{H} \text {, }
$$

which we therefore now assume. Then $\left\{C\left(\alpha_{Q}\right)\right\}^{r} \in \hat{G}$ implies that

$$
\left\{C\left(\alpha_{Q}\right)\right\}^{r} \in \hat{H} \text {. }
$$

Here, as usual, $\hat{H}$ is the group generated by the classes $\left\{C^{*}\left(\alpha_{Q}\right)\right\}$ of $(3)$, with $q=q(Q)$. We saw in $\S 1$ that (12) is a necessary and sufficient condition for the induced projection mapping from $\hat{F}$ into $F$ to be a monomorphism.

Let $\tilde{R}$ be the simply connected smooth universal covering surface of $R$, and let $\tilde{C}$ be a curve in $\tilde{R}$ lying over the curve $C\left(\alpha_{Q}\right)$ and joining a point $\tilde{A}$ over $A$ to itself ; $\tilde{C}$ is closed since $C\left(\alpha_{Q}\right)$ is homotopic to a constant in $R$. The end point $\tilde{Q}^{\prime}$ of the portion $\tilde{\alpha}_{Q}$ of $\tilde{C}$ which lies over $\alpha_{Q}$ lies over $Q^{\prime}$, and determines uniquely a point $\tilde{Q}$ over $Q$ which lies in the same open disk $\tilde{N}_{Q}$ over $N_{Q}$ as $\tilde{Q}^{\prime}$ does. Let $\vec{R}_{Q}$ and $\tilde{R}_{Q}$ be the manifolds obtained from $\tilde{R}$ by removing $\tilde{Q}$, and all points lying over $Q$, respectively. Then $\tilde{R}_{Q}$ is a smooth unlimited covering surface of $R_{Q}=R-Q$, and it follows from (13) and the monodromy theorem that $\tilde{C}^{r}$ is homotopic in $\tilde{R}_{Q}$ to a product of $q(Q)$ th powers of curves $\tilde{C}\left(\beta_{Q}\right)$ lying over certain curves $C\left(\beta_{Q}\right)$. We deduce that $\tilde{C}^{r}$ is homotopic in $\bar{R}_{Q}$ to a product of $q(Q)$ th powers of curves $\tilde{C}\left(\beta_{Q}\right)$ for which $\beta_{Q}$ is homotopic to $\alpha_{Q}$ in $R$. We now use the fact that $\tilde{R}$ is homeomorphic either (i) to $Z$, or (ii) to the finite $z$-plane $Z^{\prime}$. In this homeomorphism the curves $\tilde{C}$ and $\tilde{C}\left(\beta_{Q}\right)$ map into curves encircling the image point of $\tilde{Q}$, and it follows immediately in case (ii) that $r$ is divisible by $q(Q)$, so that (11) holds.

There remains the case (i) when $\tilde{R}$ is homeomorphic to $Z$. Then $R$ is also homeomorphic to $Z$. Suppose first that $B$ contains three or more points, and let $Q_{1}=Q, Q_{2}$ and $Q_{3}$ be three of them. Write $q_{i}=q\left(Q_{i}\right)(i=1,2,3)$, and let $R^{\prime}$ be a branched covering surface of $R$ having branch points of orders $q_{i}-1$ over the points $Q_{i}(i=1,2,3)$. For this purpose we may take $R^{\prime}$ to be the Riemann surface of the associated Riemann-Schwarz triangle function. The curves in $R^{\prime}$ lying over the curves $\left[C\left(\alpha_{Q_{i}}\right)\right]^{q_{i}}$ are closed, so that (13) implies that the curves lying over $C^{r}\left(\alpha_{Q}\right)$ are closed, and it follows that $q(Q)=q_{1}$ divides $r$. Hence (11) holds.

If, however, $B$ contains only two points $Q_{1}=Q$ and $Q_{2}$, then $H$ is the infinite cyclic group generated by $\left\{C\left(\alpha_{Q}\right)\right\}$, since $C\left(\alpha_{Q_{1}}\right) C^{ \pm 1}\left(\alpha_{Q_{2}}\right)$ is homotopic to a constant in $R-Q_{1}-Q_{2}$. Thus $H$ is generated by the $q\left(Q_{1}\right)$ th and $q\left(Q_{2}\right)$ th powers of $\left\{C\left(\alpha_{Q}\right)\right\}$, and so we can conclude from (13) that $q(Q)$ divides $r$, if and only if $q\left(Q_{1}\right)=q\left(Q_{2}\right)$.

Finally, if $B$ contains only one point, every $C\left(\alpha_{Q}\right)$ is homotopic to a constant, and so (11) is not true. We have therefore proved

Theorem 2. If, in the notation of Theorem 1 , the group $G$ is such that

$$
\hat{G} \cap H=\hat{H} \text {, }
$$

then (11) holds, except when $R$ is homeomorphic to $Z$ and either (i) $B$ consists of a single point, or (ii) $B$ consists of two points $Q_{1}$ and $Q_{2}$, and $q\left(Q_{1}\right) \neq q\left(Q_{2}\right)$. Except in these cases, therefore, regular branched covering surfaces of the required type exist and can be constructed. In particular, 
an unlimited regular branched simply connected surface $\hat{R}$ can be constructed except in the two cases mentioned above.

We note that regular branched covering surfaces exist for which (12) does not hold. Thus the Riemann surface $\hat{R}$ of the algebraic equation

$$
w^{2}=\left(z-e_{1}\right)\left(z-e_{2}\right)\left(z-e_{3}\right)\left(z-e_{4}\right)
$$

with distinct $e_{i}(i=1,2,3,4)$ is a regular branched covering surface of $R=Z$. Closed curves in $R$ encircling exactly two branch points once determine a class of curves of $\hat{G}$ which belongs to $H$ but not to $\hat{H}$.

4. General branched covering surfaces. In $\S 3$ our object was to construct a regular covering surface $\hat{R}$ over $R$ with branch points of given orders; i.e. we started from a set of numbers $q(Q)(Q \in B)$ which we used to construct the group $\hat{H}$, and this limited our choice of the group $\hat{G}$. The problem is, however, in many ways simpler if we adopt a different point of view.

We start from a Riemann surface $R$ with a closed set $B$ of isolated points on it and define $S, F, G$ and $H$ as before. We now take any subgroup $\hat{G}$ of $G$ which is such that it contains some positive power of each class $\left\{C\left(\alpha_{Q}\right)\right\}(Q \in B)$, and define $q\left(\alpha_{Q}\right)$ to be the least positive exponent of $\left\{C\left(\alpha_{Q}\right)\right\}$ for which this property holds. It is easily seen that $q\left(\alpha_{Q}\right)=q\left(\beta_{Q}\right)$ whenever $\left\{\alpha_{Q} \gamma_{Q}^{r} \beta_{Q}^{-1}\right\} \in \hat{G}$ for some integer $r$. Let $\hat{H}$ be the subgroup generated by the classes $\left\{C\left(\alpha_{Q}\right)\right\}^{q\left(\alpha_{Q}\right)}$; clearly $\hat{H}$ is normal in $\hat{G}$.

We now construct an unlimited branched covering Riemann surface $\hat{R}$ over $R$ exactly as in $\S 3$, with $G^{\prime}$ replaced by $\hat{G}$ and $H^{\prime}$ by $\hat{H}$. The argument goes through as before and we find that $\hat{R}$ is smooth over $R$ except at the points $\hat{Q}=\left\{Q, \alpha_{Q}\right\}(Q \in B)$, where there are branch points of order $q\left(\alpha_{Q}\right)-1$. The fundamental group of the surface $\hat{S}$ is $\hat{G}$ and the fundamental group of $\hat{R}$ is isomorphic to $\hat{G} / \hat{H}$. Since $\hat{G}$ need not be normal in $G$, the covering surfaces $\hat{R}$ and $\hat{S}$ need not be regular. In particular, it is possible to construct simply connected branched covering surfaces which are not regular.

5. Infinite winding points. Branched covering surfaces having infinite (logarithmic) winding points over certain isolated points on a given Riemann surface $R^{\prime}$ can be catered for similarly merely by omitting such points before constructing the covering surface.

For suppose that it is desired to construct a regular branched covering surface $\hat{R}$ over $R^{\prime}$ which has ordinary branch points of given orders at points of $\hat{R}$ lying over a set $B$ of isolated points of $R^{\prime}$, and infinite winding points over a set $B^{\infty}$ of isolated points of $R^{\prime}$. We put $R=R^{\prime}-B^{\infty}$ and construct $\hat{R}$ as a covering surface over $R$ as described in $\S 3$. It may be noted that, when $B^{\infty}$ is not empty, $R$ cannot be homeomorphic to $Z$, so that the exceptional cases mentioned in Theorem 2 cannot arise. Similar remarks apply to the more general branched covering surfaces considered in $\$ 4$.

6. Regular branched coverings over branched coverings. We now consider the situation described in the first paragraph of $\S 1$.

We suppose that $B^{\prime}$ is a closed set of isolated points on a Riemann surface $R^{\prime}$, and that $\tilde{R}^{\prime}$ is an unlimited branched covering surface of $R^{\prime}$ with branch points of order $q(\tilde{Q})-1$ at the points $\tilde{Q}$ of $\tilde{R}^{\prime}$ which lie over points $Q$ of $B^{\prime}$. We assume that $q(\tilde{Q}) \geqslant 1$, and do not require $\tilde{R}^{\prime}$ to be regular over $R^{\prime}$. 
To each point $\tilde{Q}$ of $\tilde{B}^{\prime}$ (the set of points of $\tilde{R}^{\prime}$ lying over $B^{\prime}$ ) we assign a positive integer $r(\tilde{Q})$ in such a way that $q(\tilde{Q}) r(\tilde{Q})$ is the same for all points $\tilde{Q}$ lying over any point $Q \in B^{\prime}$; i.e.

$$
q(\tilde{Q}) r(\tilde{Q})=s(Q)
$$

say. We assume that $s(Q)>1$ as otherwise the points are ordinary points. If $\tilde{R}^{\prime}$ has infinitely many sheets and is not regular over $R^{\prime}$, it may not be possible to choose finite integers $r(\tilde{Q})$ and $s(Q)$ satisfying (14), and we denote by $B^{\infty}$ the subset of $B^{\prime}$ consisting of points $Q$ for which (14) is not possible, or for which we do not wish to assign a finite $s(Q)$. Let $\tilde{B}^{\infty}$ be the set of points of $\tilde{R}^{\prime}$ lying over $B^{\infty}$, and write

$$
\begin{array}{ll}
B=B^{\prime}-B^{\infty}, \quad R=R^{\prime}-B^{\infty}, \quad S=R-B, \\
\tilde{B}=\tilde{B}^{\prime}-\tilde{B}^{\infty}, \quad \tilde{R}=\tilde{R}^{\prime}-\tilde{B}^{\infty}, \quad \tilde{S}=\tilde{R}-\tilde{B} .
\end{array}
$$

Our object is to construct an unlimited branched covering surface $\hat{R}$ over both $R$ and $\tilde{R}$, which is regular over $R$; $\hat{R}$ will then automatically be regular over $\tilde{R}$. We wish $\hat{R}$ to have branch points of order $r(\tilde{Q})-1$ over points $\tilde{Q} \in \tilde{B}$, and branch points of order $s(Q)-1$ over points $Q \in B$. We write $\hat{S}=\hat{R}-\tilde{B}$, where $\hat{B}$ is the set of points of $\hat{R}$ which lie over $B$.

Let $G, \tilde{G}$ and $\hat{G}$ (as yet unspecified) be the fundamental groups of the surfaces $S, \tilde{S}$ and $\hat{S}$, and let $H, \tilde{H}$ and $\hat{H}$ be the subgroups generated by the classes

$$
\left\{C\left(\alpha_{Q}\right)\right\}, \quad\left\{C\left(\alpha_{Q}\right)\right\}^{\alpha(\tilde{Q})}, \quad\left\{C\left(\alpha_{Q}\right)\right\}^{s(\boldsymbol{Q})},
$$

for all $Q \in B$. Here $\tilde{Q}$ is the point over $Q$ in $\tilde{R}$ whose open disk $\tilde{N}_{Q}$ contains the end point $\tilde{Q}^{\prime}$ of the curve $\tilde{\alpha}_{Q}$ drawn over $\alpha_{Q}$ in $\tilde{S}$ from a base point $\tilde{A}$ over $A$. The group structure is shown in the diagram, normal subgroups being indicated by full lines.

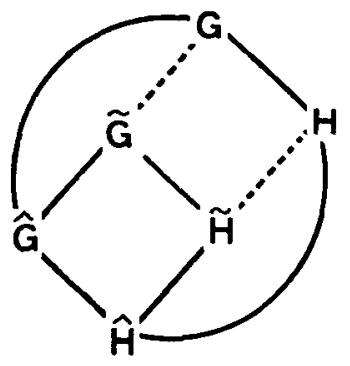

If $F, \tilde{F}$ and $\hat{F}$ are the fundamental groups of $R, \tilde{R}$ and $\hat{R}$, we have

$$
F \cong G / H, \quad \tilde{F} \cong \tilde{G} / \tilde{H}, \quad \hat{F} \cong \hat{G} / \hat{H} .
$$

We suppose that $\hat{G}$ is a normal subgroup of $G$ containing $\hat{H}$ such that

$$
\left\{C\left(\alpha_{Q}\right)\right\}^{p} \in \hat{G} \text { implies that } s(Q) \text { divides } p \text {. }
$$

This condition is necessary if $\hat{R}$ is to be regular over $R$ and have branch points of the right orders. Then $\hat{G}$ is also normal in $\hat{G}$, and so, by Theorem 1 , we can construct a regular covering surface $\hat{R}$ over $\tilde{R}$ which has branch points of order $r(\tilde{Q})-1$ at points $\hat{Q}$ lying over the points $\tilde{Q} \in \tilde{B}$, provided that

$$
\left\{\tilde{C}\left(\tilde{\alpha}_{g}\right)\right\}^{t} \in \hat{Q} \text { implies that } r(\tilde{Q}) \text { divides } t \text {. }
$$


In (16), $\tilde{C}\left(\tilde{\alpha}_{Q}\right)$ is a closed curve in $\tilde{S}$ of the form

$$
\dot{\tilde{C}}\left(\tilde{\alpha}_{Q}\right)=\tilde{\alpha}_{Q} \tilde{\gamma}_{Q} \tilde{\alpha}_{Q}^{-1},
$$

in an obvious notation, and so lies over $C^{\alpha}\left(\alpha_{Q}\right)$, where $q=q(\tilde{Q})$. Thus (16) states that we require that

$$
\left\{C\left(\alpha_{Q}\right)\right\}^{a t} \in \hat{G} \text { implies that } r(\tilde{Q}) \text { divides } t,
$$

and this is satisfied by (14) and (15).

Thus $\hat{R}$ can be constructed and is regular over $\tilde{R}$. It is also regular over $R$, since $\hat{G}$ is normal in $G$. That the branch points of $\hat{R}$ over $R$ are of the required order follows from (15) as in $\S 3$. It follows that $\hat{R}$ is a regular branched covering surface of $R$ and $\tilde{R}$ of the desired type.

In particular, a simply connected regular branched covering surface $\hat{R}$ of $R$ and $\tilde{R}$ can be constructed except in the two cases mentioned in Theorem 2. Also infinite winding points can be catered for as described in $\S \mathbf{5}$.

\section{REFERENCES}

1. L. Fourès, Sur les recouvrements regulièrement ramifiés, Bull. Sci. Math. 76 (1952), 17-32.

2. R. A. Rankin, The Schwarzian derivative and uniformization, Journal d'Analyse. 6 (1958).

3. G. Springer, Introduction to Riemann surfaces (Reading, 1957).

The UnIVERsity

Glascoow 